ARTICLE

Received 10 Dec 2014 | Accepted 9 Feb 2015 | Published 23 Mar $2015 \quad$ DOl: 10.1038/ncomms7585 OPEN

\title{
Targeted diversity generation by intraterrestrial archaea and archaeal viruses
}

\author{
Blair G. Paul', Sarah C. Bagby¹, Elizabeth Czornyj², Diego Arambula², Sumit Handa ${ }^{3}$, Alexander Sczyrba ${ }^{4,5}$,
} Partho Ghosh ${ }^{3}$, Jeff F. Miller $2,6,7$ \& David L. Valentine ${ }^{1,8}$

In the evolutionary arms race between microbes, their parasites, and their neighbours, the capacity for rapid protein diversification is a potent weapon. Diversity-generating retroelements (DGRs) use mutagenic reverse transcription and retrohoming to generate myriad variants of a target gene. Originally discovered in pathogens, these retroelements have been identified in bacteria and their viruses, but never in archaea. Here we report the discovery of intact DGRs in two distinct intraterrestrial archaeal systems: a novel virus that appears to infect archaea in the marine subsurface, and, separately, two uncultivated nanoarchaea from the terrestrial subsurface. The viral DGR system targets putative tail fibre ligand-binding domains, potentially generating $>10^{18}$ protein variants. The two single-cell nanoarchaeal genomes each possess $\geq 4$ distinct DGRs. Against an expected background of low genomewide mutation rates, these results demonstrate a previously unsuspected potential for rapid, targeted sequence diversification in intraterrestrial archaea and their viruses.

\footnotetext{
${ }^{1}$ Marine Science Institute, University of California, Santa Barbara, California 93106, USA. ${ }^{2}$ Department of Microbiology, Immunology and Molecular Genetics, University of California, Los Angeles, California 90095, USA. ${ }^{3}$ Department of Chemistry and Biochemistry, University of California San Diego, La Jolla, California 92093, USA. ${ }^{4}$ Center for Biotechnology and Faculty of Technology, Bielefeld University, 33615 Bielefeld, Germany. ${ }^{5}$ DOE Joint Genome Institute, Walnut Creek, California 94598, USA. ${ }^{6}$ Molecular Biology Institute, University of California, Los Angeles, California 90095, USA. ${ }^{7}$ California NanoSystems Institute, University of California, Los Angeles, California 90095, USA. ${ }^{8}$ Department of Earth Science, University of California Santa Barbara, Santa Barbara, California 93106 USA. Correspondence and requests for materials should be addressed to D.L.V. (email: valentine@geol.ucsb.edu).
} 


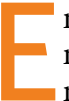
nergy-limited marine and terrestrial subsurface environments harbour a microbial reservoir of exceptional magnitude ${ }^{1}$. Archaea are both numerically dominant ${ }^{2}$ and well adapted to energy limitations faced in various intraterrestrial environments ${ }^{3,4}$. Although little is understood about their physiology, metabolism, evolution, or mortality in these environments, current research predicts that they will be characterized by slow growth and low genome-wide mutation rates $^{5}$.

Independent of the sporadic mutation rate, microbial genetic variation can be increased by processes such as gene conversion and horizontal gene transfer. The single most powerful such mechanism known in nature is the diversity-generating retroelement (DGR $)^{6,7}$. DGRs use a process called mutagenic retrohoming for the targeted replacement of a variable repeat (VR) coding region with a sequence derived from reverse transcription of a cognate non-coding template repeat (TR) $\mathrm{RNA}^{6-9}$. Crucially, the reverse transcriptase (RT) used is errorprone at template adenine bases ${ }^{10}$, but has high fidelity at other template bases, modulating the rate of diversification to permit rapid exploration of target protein (TP) variants within a recognizable structural framework. Over successive waves of replication, DGR activity leads to rapid evolution of TPs, typically altering ligand-binding specificity ${ }^{11}$ and even permitting phage recognition of novel host ligands 9 . To date, DGRs have been found widely in bacteria and their viruses, but never in an archaeal system.

Because parasitism is expected to be an important driver of evolution and mortality in intraterrestrial archaea ${ }^{12}$, we set out to identify and characterize viruses of anaerobic archaea from one system in the marine subsurface, a methane seep in a California borderlands basin. Our survey uncovers the complete genome of a virus that appears to infect archaea. Remarkably, this genome encodes a complete and apparently active DGR. We examine existing sequence data from archaeal systems, discovering multiple DGRs in the genomes of two subterranean nanoarchaea. These findings demonstrate that subsurface archaea and archaeal viruses maintain a mechanism for generating protein hypervariability within targeted genes, bringing the capacity for massive diversification to the archaea-dominated deep biosphere.

\section{Results}

A putative archaeal virus encodes a DGR. We collected subsurface sediments from a methane seep at $820 \mathrm{~m}$ water depth in Santa Monica Basin. After confirming that these sediments exhibited anaerobic oxidation of methane (Supplementary Fig. 1), we prepared and sequenced a viral metagenome, uncovering a novel and apparently complete viral genome (termed ANMV-1; Fig. 1a). Examination of ANMV-1 coding sequences offered two key lines of evidence that this virus infects an archaeal host. First, the ANMV-1 genome encodes a TATA-box binding protein, an essential component of the transcriptional machinery in archaea and eukarya that is absent from bacteria ${ }^{13}$. Second, the ANMV-1 genome contains six genes that show sequence similarity ( $e$-value $10^{-7}$ to $10^{-26}$ ) with proteins from methanotrophic archaea (ANME-1 and ANME-2D) and none with comparable similarity to eukaryotic proteins (Supplementary Table 1). We further hypothesize that ANMV-1's archaeal host is anaerobic; ribonucleotide reductase activity is essential for phage genome replication $^{14}$, and ANMV-1 encodes an oxygen-sensitive ribonucleotide reductase. In light of the active anaerobic oxidation of methane metabolism observed in the sample from which ANMV-1 was sequenced, the anaerobic archaeal host may belong to an anaerobic methane-oxidizing (ANME) clade.

Analysis of ANMV-1 identified a cassette bearing a RT gene, two 114-bp proximal repeats that vary from each other at positions corresponding to adenines, and a short inverted repeat with potential for hairpin formation (Fig. 1b). Together, these features are hallmarks of a $\mathrm{DGR}^{6-9}$. Since the discovery of these remarkable elements, $>300$ DGRs have been identified, all within the bacteria and their viruses ${ }^{15,16}$. ANMV-1 represents the first identification of a DGR that appears to operate in an archaeal system.

Although the ANMV-1 VR lies within a gene of unknown function (best BLASTp $e$-value $>10^{-3}$, to uncharacterized proteins), the predicted secondary structure of the gene product

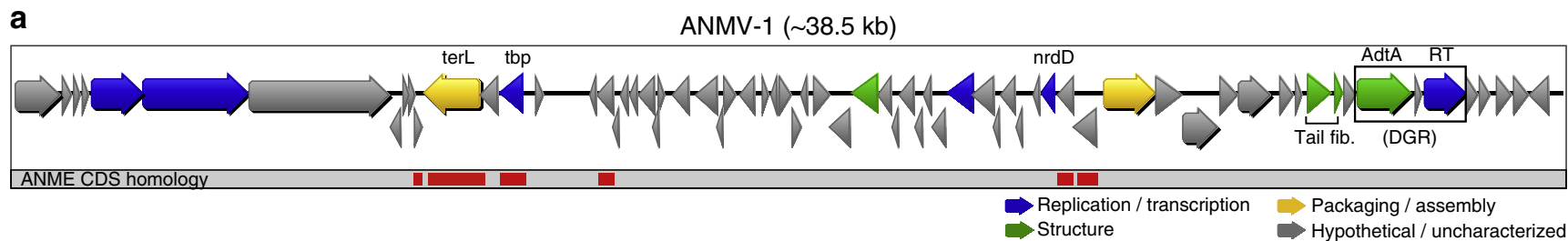

b

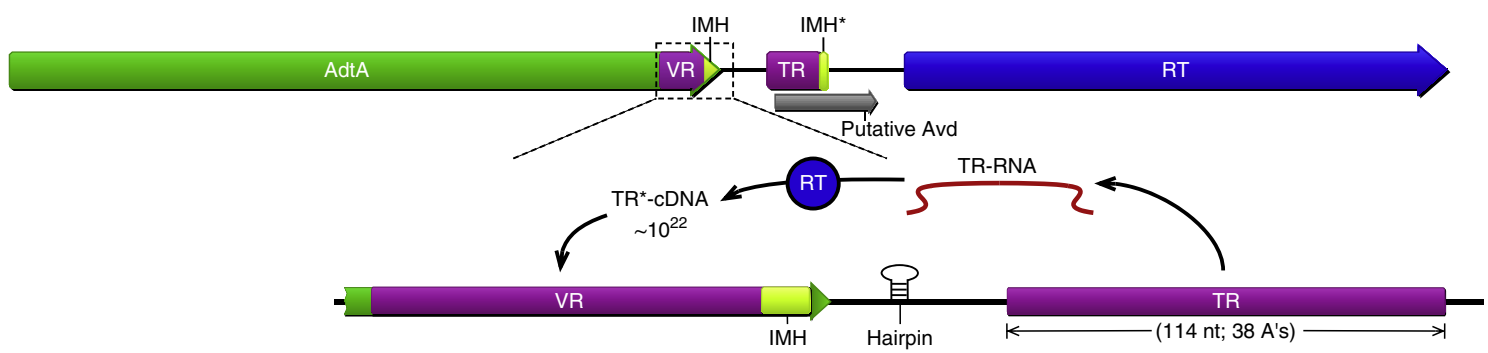

Figure 1 | Retroelement-containing ANMV-1 genome obtained from methane seep sediment. (a) Annotated coding sequences (CDS) designated by arrows that are coloured according to predicted function. Genes with blast similarity to ANME protein sequences are highlighted in red below each corresponding ANMV-1 locus (Supplementary Table 1). Symbols above selected annotations indicate putative gene names: terL, terminase large subunit; tbp, TATA-box binding protein; $\mathrm{nrdD}$, anaerobic ribonucleoside triphosphate reductase; AdtA, DGR TP; RT, reverse transcriptase. An open box highlights the DGR cassette with flanking putative tail fibres (tail fib.), shown below the genome. (b) Putative cis- and trans-acting features of the ANMV-1 DGR. RT, accessory variability determinant (Avd) and AdtA ORFs are shown as blue, grey and green arrows, respectively. Purple boxes indicate template and variable repeat regions ( $T R$ and $V R$ ). The IMH and cognate $I M H^{\star}$ sites are highlighted in yellow. The expanded DGR view depicts the putative retrohoming target site. Estimated number of nucleotide sequence variants is given above VR (TR* $c D N A s)$, based on theoretical mutagenesis of adenines in TR intermediate RNA. 
a DUSEL4 nanoarchaeota OTU1
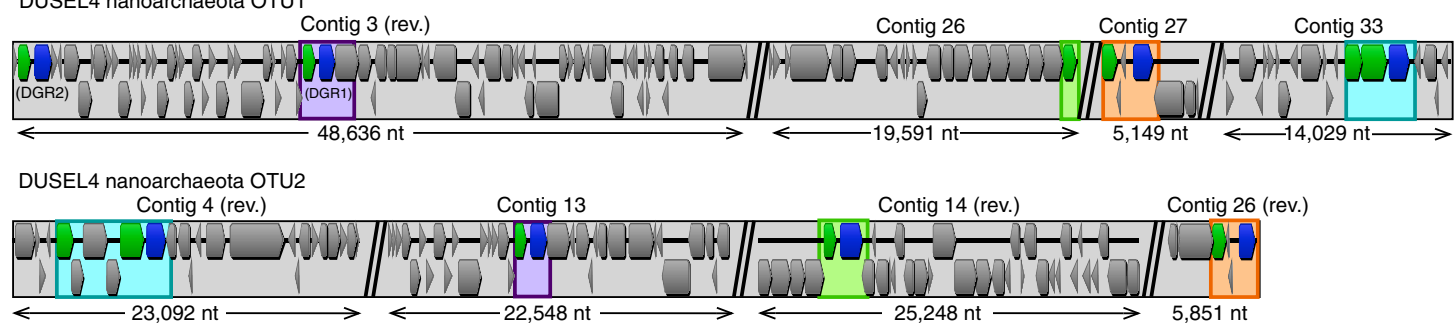

b

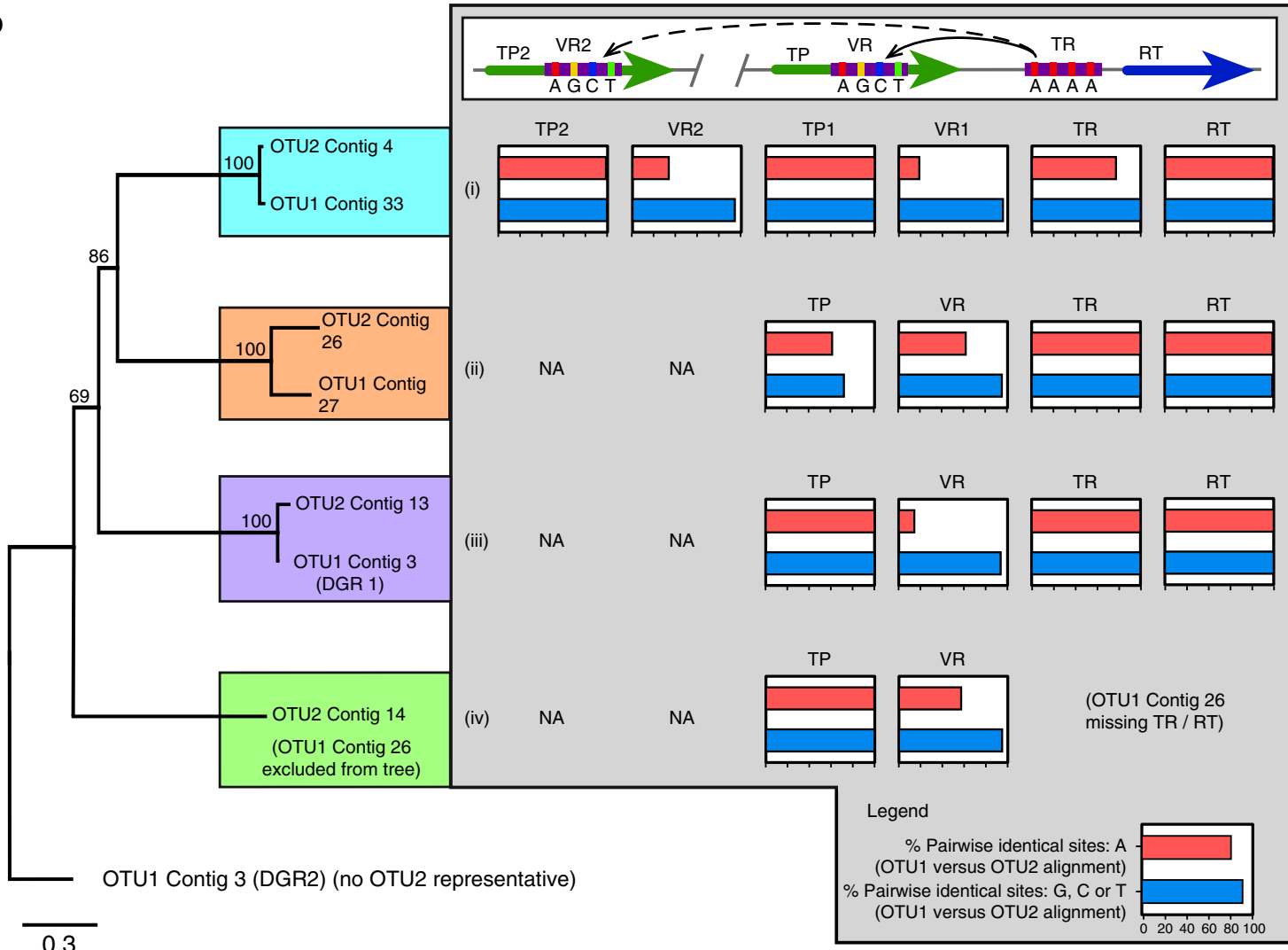

Figure 2 | Grouping of DGRs from Nanoarchaeota. (a) Positions of four DGR cassettes in each OTU, coloured by homology-based groups (note ungrouped OTU1 DGR in grey). Contigs are shown with DGRs on the forward strand (rev., reverse complement). (b) DGR groups, ordered by RT and TP homologies. A PhyML tree (left) was constructed with 100 bootstrap replicates (support indicated on branches) from concatenated alignments of TP and RT amino-acid sequences for each complete DGR cassette. Group 4 includes an incomplete DGR for OTU1 contig 26 (missing RT ORF). A schematic for nanoarchaeal DGRs shows the direction of information transfer during targeted mutagenesis. TP and RT genes are shown as green and blue arrows, respectively, while purple boxes indicate variable and template regions (VR and TR). Bar graphs show pairwise similarity between aligned OTU1 and OTU2 sequences for major DGR features, TP, VR, TR and RT. NA (not applicable) indicates that a feature is not found in the DGR.

offered important functional insights. The ANMV-1 DGR target (termed AdtA) shares greatest structural homology (37\% of residues modelled with 99\% Phyre confidence; r.m.s.d. $1.6 \AA$ $Z=13.6)$ with the major tropism determinant (Mtd) of Bordetella phage BPP-1, a DGR-targeted tail fibre protein responsible for binding host ligands. AdtA contains 21 codons with potential for adenine-specific amino-acid substitutions (versus 12 in $\mathrm{Mtd}$ ), including nine AAY codons, with potential for $>10^{18}$ variants. Thus, ANMV-1 demonstrates a degree of coding variability that is comparable to bacterial DGR systems ${ }^{11}$ and outpaces the vertebrate immune system's capacity to generate variants of antibodies or T-cell receptor proteins ${ }^{17,98}$. Predicted AdtA structural homology to Mtd is greatest in its $\mathrm{C}$ terminus, which corresponds to the C-type lectin (CLec)-fold common to many known bacterial DGR targets ${ }^{11,15}$. As in Mtd, the targeted AdtA residues map to partially solvent-exposed sites in the CLec domain (Supplementary Fig. 2). Together, these findings point to a binding-related role for AdtA, and the genomic proximity of the adtA gene to phage tail fibre genes (Fig. 1a) suggests host attachment as a possible function.

The discovery of a mechanism for rapid genetic diversification in ANMV-1 raises questions about the distribution and evolution of this virus. We conducted a search for close relatives of the ANMV-1 genome in environmental metagenomic databases, identifying a group of highly similar sequences (Supplementary Fig. 3) found in seafloor sediments of the Nyegga methane seeps, offshore Norway ${ }^{19}$, and in Coal Oil Point hydrocarbon seeps, offshore Santa Barbara, California. Metagenomes from both seeps cover portions of the ANMV-1 DGR cassette, including a closely related and intact RT open reading frame (ORF) from Nyegga seep sediments. These results indicate that ANMV-1 relatives are widespread in methane seeps. Furthermore, the persistence of 
Group

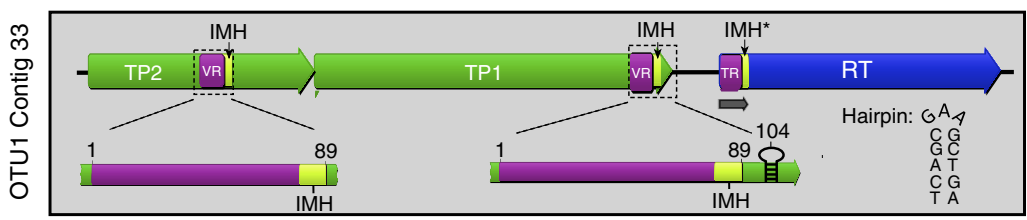

(ii)

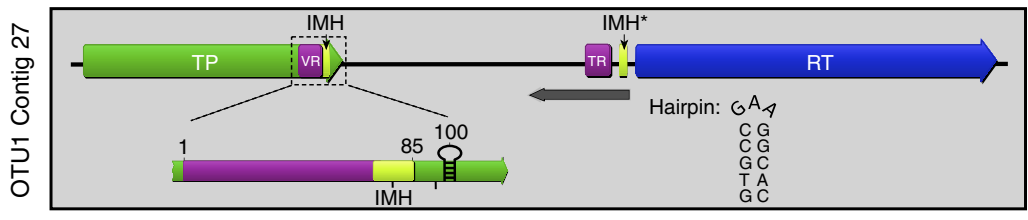

(iii)

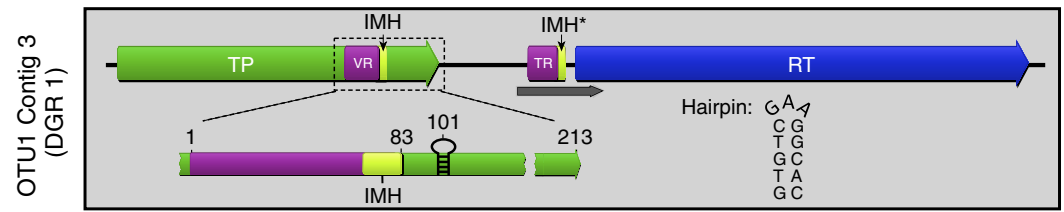

(iv)
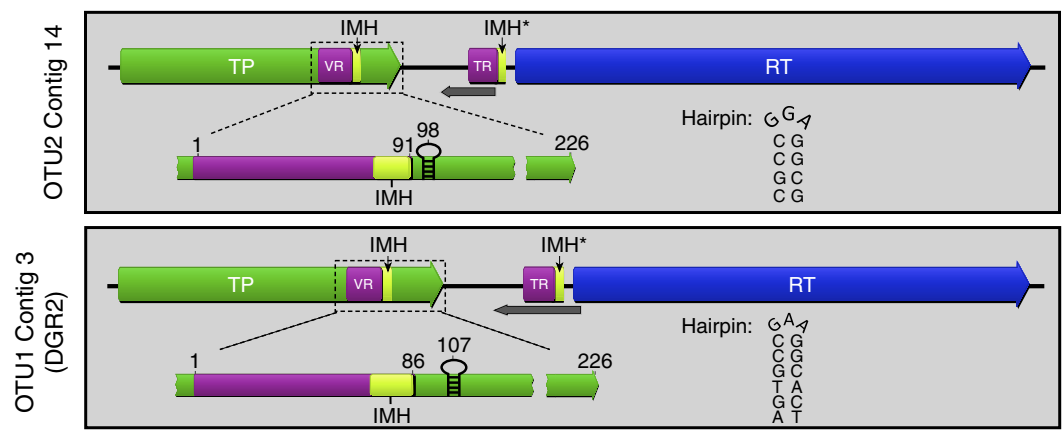

Figure 3 | Conserved and putative regulatory features of Nanoarchaeota DGRs. IMH sites $\left(\mathrm{IMH}\right.$ and $\left.\mathrm{IMH}^{\star}\right)$ are shown as yellow boxes, and the trinucleotide-loop hairpin is given in an expanded view at right. Dark grey arrows indicate ORFs between RT and TP whose amino-acid sequences have comparable isoelectric point and molecular weight to accessory variability determinant (Avd; $\mathrm{pl}=9 \pm 1 ; M_{\mathrm{w}}=10 \pm 5$ ).

DGR sequences in related viruses from widely separated ocean basins suggests a selective pressure to maintain the mechanism for targeted protein diversification.

Two Nanoarchaeota maintain multiple DGRs. Having identified the first DGR-containing archaeal system, an apparently widespread virus from the marine subsurface, we asked whether distinct DGRs might occur in intraterrestrial archaea themselves. We searched genomic databases for archaeal RT genes and nearby repeats with adenine variability, finding multiple putative DGRs in the two operational taxonomic units (OTU1 and OTU2) of DUSEL4, a clade of uncultivated subterranean Nanoarchaeota established from four sequenced cells ${ }^{20}$. Whereas the sequenced genomes of the other known nanoarchaea, Nanoarchaeum equitans $^{21}$ (completely sequenced) and Nanoarchaeote Nst-1 (ref. 22) ( $\sim 91 \%$ sequenced), so far appear to contain neither DGRs nor RT genes, the DUSEL4 genomes have an abundance, with four distinct (non-redundant) DGR cassettes in a single genome (Fig. 2a). Examination of DUSEL4 RT and TP sequences revealed four distinct groups of DGRs with conserved cis- and trans-acting features, each with a single representative in both OTU1 and OTU2 (Figs 2b and 3). Intriguingly, a further search within these genomes for VR-containing genes revealed two partial DGRs - consisting only of a target gene, VR, and cis-acting elements-in OTU1, the representative with higher estimated genome coverage ${ }^{20}$. Evidence of adenine-directed mutagenesis in these VRs (Supplementary Fig. 4) suggests a history of DGR activity in these sites that do not contain an RT gene, indicating either that the fragments are fossils, left behind when the RT was recruited to a different genomic location or simply lost, or that they are diversified remotely by DGRs elsewhere in the genome.

Archaeal DGR components have distinct evolutionary histories. The possibility that DGRs might not move as a unit led us to examine the evolutionary histories of key DGR cassette components. First, we analysed the phylogeny of the newly identified archaeal DGR RTs. Canonical DGR-type RTs have been shown to form a distinct clade most closely related to bacterial group-II introns ${ }^{7,23,24}$; while known archaeal RTs are most similar to bacterial group-II and group-II-like introns, they fall outside the DGR clade ${ }^{24}$. We find that the RTs from ANMV-1 and DUSEL4 DGRs lie in a monophyletic group within the DGR clade (Fig. 4a), branching separately from bacterial sequences ( $97 \%$ bootstrap support; Fig. 4b). Underscoring the likelihood that ANMV-1 has an archaeal host, this pattern suggests that ANMV-1 and DUSEL4 DGR RTs share a common archaeal ancestry.

We next compared the tetranucleotide composition of DUSEL4 DGRs to that of their host genomes (for individual genome signatures, see Supplementary Fig. 5) at two levels: the concatenated DGRs, and separately concatenated DGR TP genes and RT genes. While TP fragments lie well within the core genomic pattern, RT fragments present as outliers, pulling the overall DGR signature away from the genome core (Fig. 5a,b). Together with the RTs' phylogenetic relationships, this pattern suggests that DUSEL4 may have acquired its DGR RTs via horizontal transfer, perhaps from another archaeal host. The sequence conservation 
a

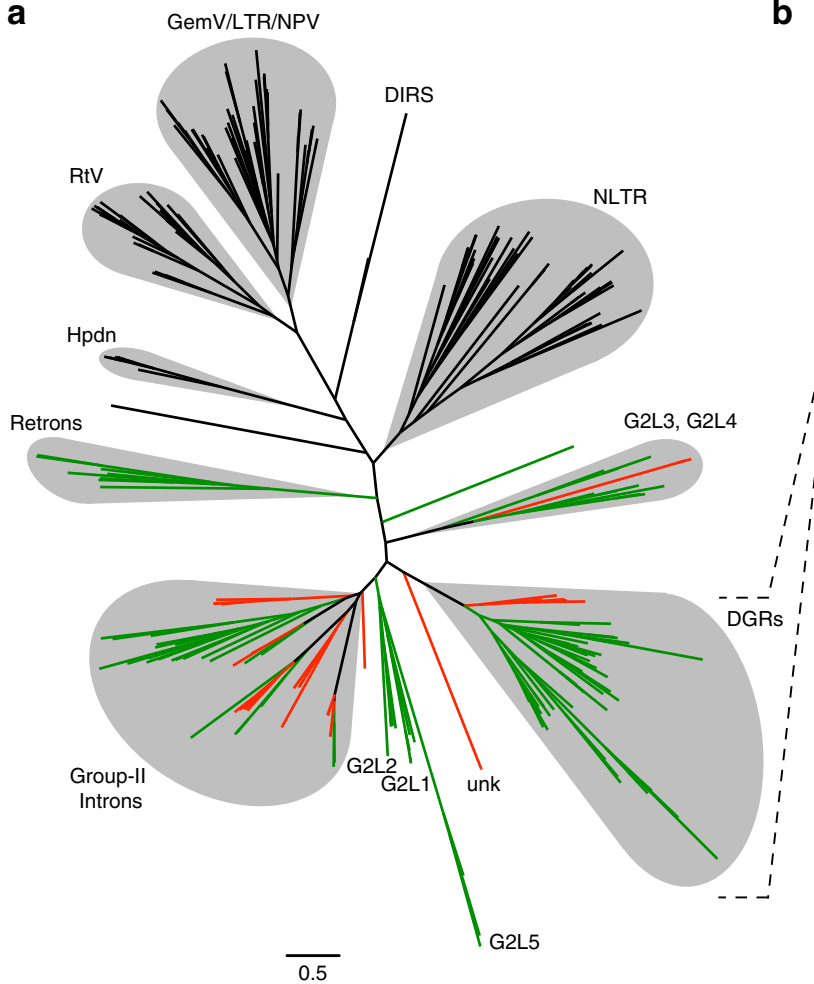

b

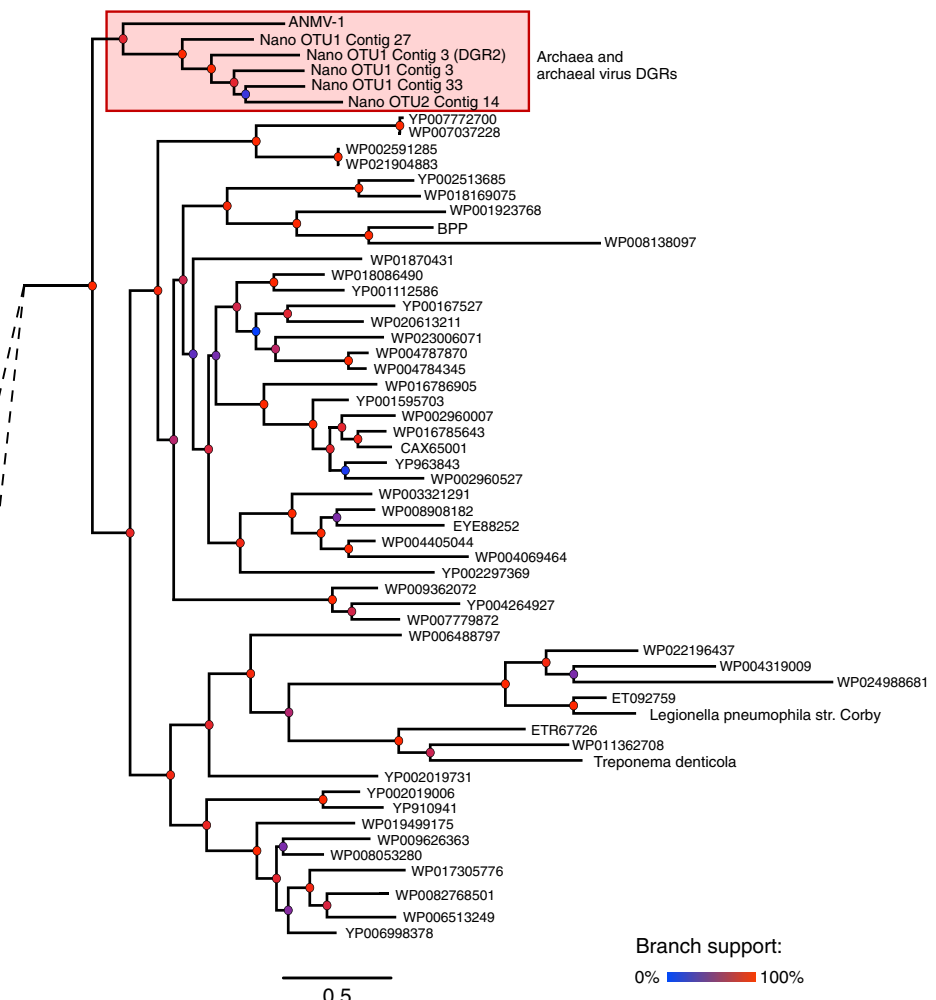

Figure 4 | RT phylogeny for archaeal DGRs. (a) Maximum-likelihood phylogenetic tree of RT representatives aligned with ANMV-1 and DUSEL4 Nanoarchaeota sequences. Green branches correspond to bacterial and bacteria-derived RTs (from chromosomes, plasmids, mitochondria, chloroplasts and bacteriophage), red branches indicate archaeal and archaeal virus RTs, and black branches represent RTs from eukaryotes and their viruses. Retroelement clades and key representatives are labelled as follows: DGRs, diversity-generating retroelements; DIRS, Dictyostelium retrotransposons; GemV, geminiviridae; G2L, group-II intron-like (G2L are numbered according to Simon and Zimmerly (24)); Hpdn, hepadnaviruses; LTR, long terminal repeat retroelements; NPV, nucleopolyhedralviruses; non-LTR, non-long terminal repeat retroelements; RtV, retroviridae; unk, unknown or unclassified. The scale shows substitutions per site. For clarity, bootstrap values are not shown for the full RT tree. (b) Expanded subtree view of DGR RT representatives. A red box highlights the archaeal DGR clade. NCBI accession codes are given for representatives in the subtree, but previously described bacterial DGRs are explicitly named. The representative for Bordetella phage BPP is labelled 'BPP'. Coloured circles at internal nodes indicate branch support.

across multiple DGR RTs in DUSEL4 (Supplementary Fig. 6a) suggests that they have a common source, perhaps a single acquisition followed by repeated gene duplications as new DGRs formed.

Nanoarchaeal DGRs target orphan genes. Most previously identified bacterial and phage DGRs diversify ligandbinding proteins, predominantly C-type lectin-like ${ }^{9,11,95}$ or immunoglobulin-like folds ${ }^{23,25}$. By contrast, primary sequence analysis of all DUSEL4 Nanoarchaeota DGR and DGR fragment TPs reveals that they share no protein sequence homology with either AdtA or any database representatives, but rather constitute a set of orphan genes (Supplementary Fig. 6b); this finding is supported by Phyre analysis, which predicted no structural homology between characterized proteins and any nanoarchaeal TP. Initial structural investigation of one nanoarchaeal TP (OTU1 contig 3 DGR2 TP; Fig. 2b) by circular dichroism (CD) revealed that the purified protein adopts a thermostable fold $\left(T_{\mathrm{m}} \sim 70^{\circ} \mathrm{C}\right.$; Supplementary Fig. 7$)$ even with limited secondary structure $(12 \% \alpha \text {-helix and } 25 \% \beta \text {-strand })^{26}$. Pairwise sequence alignments of the nanoarchaeal TPs (Supplementary Fig. 6b) suggest that the targets of groups i-iv are unlikely to share substantial structural homology with each other, raising the possibility that nanoarchaeal DGRs may target a broader range of protein activities than are known for bacterial and phage DGRs.

\section{Discussion}

Comparison of the putative archaeal DGRs with the canonical bacterial and viral DGRs reveals both similarities and distinctive features that may influence DGR function. In Bordetella phage BPP-1, certain cis-acting elements appear critical for efficient retrohoming, including (1) an initiation of mutagenic homing (IMH) motif that lies at the $3^{\prime}$ end of VR and an $\mathrm{IMH}^{\star}$ homologue at the $3^{\prime}$ end of TR; and (2) a short inverted repeat downstream of VR, capable of forming a hairpin/cruciform structure, typically with a GRNA tetraloop ${ }^{10}$. DUSEL4 DGRs appear to maintain versions of these canonical cis- acting elements under additional constraints. First, IMH sites in DUSEL4 include a TGGGGT motif, while DUSEL4 IMH* sites carry a corresponding TGGAAT. Second, all DUSEL4 DGR hairpins have highly constrained GRA trinucleotide loops, and each hairpin lies within its DGR's TP gene, placing this region under selection at the level of both protein structure and DNA sequence. Investigation into the influence of these features on archaeal DGR activity may shed light on differences in the molecular mechanism of DGR retrohoming in bacterial and archaeal systems.

Examination of nanoarchaeal TRs suggests the capacity for individual DGRs to generate $7 \times 10^{10}$ to $9 \times 10^{12}$ variants of their TPs, with no risk of nonsense mutations (Supplementary Fig. 4). Although this range is low by comparison with typical bacterial and viral DGRs, the potential evolutionary impact must be 

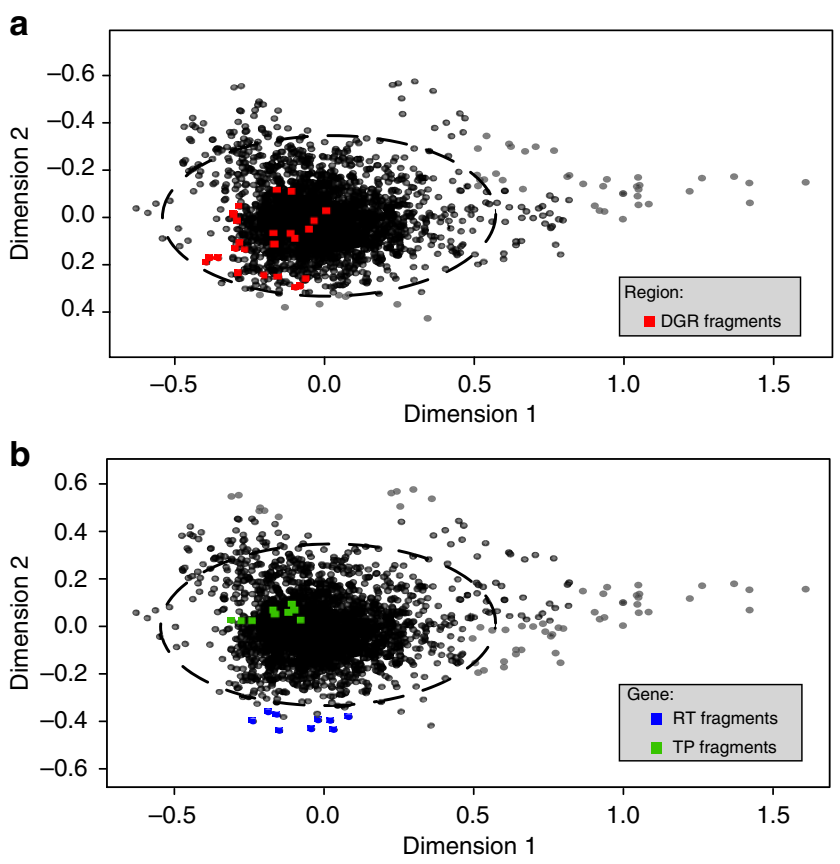

Figure 5 | Tetranucleotide distributions of DUSEL4 Nanoarchaeota.

$(\mathbf{a}, \mathbf{b})$ Non-metric multidimensional scaling plots of tetranucleotide distributions of (a) concatenated DUSEL4 DGRs (red) and (b) separately concatenated DUSEL4 DGR RT (blue) and TP genes (green), compared with the rest of the DUSEL4 Nanoarchaeota OTU1 and OTU2 genomes (greyscale circles). Each point on the ordination plots represents one 5-kb fragment. Dashed ellipses indicate the $95 \%$ confidence region.

considered in light of the multiplicity of DGRs in DUSEL4 Nanoarchaeota; whereas no bacterial or viral genome has been found to harbour $>2$ distinct DGRs, these nanoarchaea have $\geq 4$. This profusion may enable subterranean nanoarchaea to explore a multidimensional fitness landscape far more rapidly than would sporadic mutation at the low rates observed for other intraterrestrial microbes ${ }^{5}$. Moreover, the fragmentary DGRs elsewhere in OTU1 suggest either that a single nanoarchaeal DGR can concurrently target multiple genes with homologous VRs, or that these DGRs are dynamic, with mobile RT/TR elements recruited from one locus to another over time. In either case, the diversity of nanoarchaeal DGR target sequences so far discovered raises the possibility that these organisms have used DGRs as a general tool for protein engineering-a hint that scientists might be able to do the same.

It is striking that these first discoveries of DGRs in archaeal systems should occur in a virus and in the Nanoarchaeota, a phylum associated with parasitism ${ }^{21,22}$. Whether the uncultivated organisms represented by the DUSEL4 clade live as obligate parasites remains to be determined; their more important commonality with ANMV-1 may be their occurrence in Earth's subsurface. While massive and low-risk protein diversification offers clear advantages to any organism caught up in the Red Queen's race, the occurrence of a DGR in the globally distributed virus ANMV-1 and the proliferation of DGRs in subterranean nanoarchaea suggests that these elements may confer additional selective advantages in a compartmentalized and energy-limited subsurface environment.

\section{Methods}

Study site and sampling. Paull's Pingo is a seafloor mound feature (latitude $33.799^{\circ} \mathrm{N}$ and longitude $118.646^{\circ} \mathrm{W}$, depth $\sim 820 \mathrm{~m}$ ) formed by the expansion of subsurface methane hydrate ${ }^{27}$. We accessed active methane seeps at the pingo to collect sediment cores using deep submergence vehicle Alvin, during R/V Atlantis
Leg AT15-53 (September 2009). Sediment core processing was conducted shipboard in an anaerobic chamber, flushed with a nitrogen headspace. One sediment core was subsectioned between 5 and $15 \mathrm{~cm}$ (relative to seafloor) and dedicated to methane-amended incubations. Two subsamples of $60 \mathrm{ml}$ sediment were homogenized with $20 \mathrm{ml}$ of sterile, anoxic artificial seawater medium ${ }^{28}$. Incubations with the homogenized sediments were prepared in $120-\mathrm{ml}$ serum vials, under a 40 -ml headspace of $\sim 3 \% \mathrm{CH}_{4}$ and $97 \% \mathrm{~N}_{2}$. Incubations were amended with ${ }^{13} \mathrm{C}$-labelled methane (99 atom- $\%{ }^{13} \mathrm{C}$ ) as an exogenous tracer to track methane oxidation (Supplementary Fig. 1). Stable isotope ratios $\left(\delta^{13} \mathrm{C}\right)$ for $\mathrm{CO}_{2}$ were measured by isotope ratio mass spectrometry (Thermo Finnigan Delta XP Plus in continuous flow mode). After 1 month of enrichment, the incubation was terminated and viruses were purified for DNA sequencing.

Virome purification and DNA sequencing. Incubation slurry samples (1:2 sediment:aqueous phase) were used for virus particle purifications. Samples were vigorously homogenized by vortexing $(15 \mathrm{~min})$, followed by centrifugation $(10 \mathrm{~min}$ $500 \mathrm{~g})$. Supernatant was filtered $(0.22 \mu \mathrm{m})$ to separate viruses from cells. Viruses were concentrated and viral DNA was extracted as previously described ${ }^{29}$. Briefly, virus particles were concentrated via caesium chloride density gradient ultracentrifugation $\left(2 \mathrm{~h}, 22,000 \mathrm{~g}, 4^{\circ} \mathrm{C}\right)$ and treated with DNase-I. DNA was extracted by cetrimonium bromide (CTAB)-chloroform and phenol-chloroform separation. Before viral DNA amplification, a 16S PCR assay to screen for cellular DNA contamination was performed with universal bacterial primers Bact27F (5'-AGAGTTTGATCCTGGCTCAG-3') and Bact1492R (5'-GGTTACCTT GTTACGACTT-3'). Following this check, we performed Phi29 polymerase multiple displacement amplification (MDA) using the Illustra Genomiphi HY DNA Amplification Kit (GE Healthcare). Thermal cycling steps for denaturing template DNA, polymerase amplification, and post-amplification enzyme inactivation were performed according to the manufacturer's specifications, except that the MDA amplification reaction was incubated for $2 \mathrm{~h}$ instead of $4 \mathrm{~h}(2 \mathrm{~h}$, $30^{\circ} \mathrm{C}$ ). Amplified product was pyrosequenced on 454 -titanium plates at the Broad Institute, as part of the Moore Marine Phage Metagenome Initiative ${ }^{30}$. Metagenomic reads can be obtained under the NCBI BioSample accession code PRJNA47435.DV-ANM1.

Read preprocessing, binning, and assembly. Raw sequencing reads were first scanned for sequencing primers, which were identified and removed using $\mathrm{TagCleaner}^{31}$. The reads were then preprocessed to remove low-quality sequence following the method of Hurwitz et al. ${ }^{32}$, using a custom R script. Preprocessing included, first, removal of any reads with ambiguous $(\mathrm{N})$ bases; second, removal of the shortest $2.5 \%$ and longest $2.5 \%$ of reads; third, removal of reads with mean quality score $>2$ s.d. below the mean; and finally, de-replication with CD-Hit 454 (ref. 33).

Reads that passed preprocessing and quality control $(\mathrm{QC})$ steps were subjected to de novo assembly using CAMERA's meta-assembler ${ }^{34}$. As this assembler does not permit user manipulation of read overlap parameters, we compared the metaassembler output with a custom reassembly approach using Geneious v7.0 (Biomatters Ltd) with the following parameters: minimum overlap 35 bases, overlap pairwise identity $90 \%$ and index word length 12 nt. The ANMV-1 contig described in this study was generated from the meta-assembly and aligned globally with $97.7 \%$ pairwise nucleotide similarity to a contig obtained by the second custom de novo assembly. PCR screening confirmed the authenticity of the ANMV-1 DGR cassette in both template and MDA-amplified viral DNA, using primers that partially overlap TP, RT and VR/TR regions: ANMVdgrF (5'-AGGCGATGCAGACGAATGGC-3') and ANMVdgrR (5'-TTGCCCAGA GTTACACCGAGCG-3').

Metagenome annotations. Prediction of open reading frames was performed using Glimmer3 (ref. 35) with default parameters. Translated ORF sequences were annotated via CAMERA-HMM and BLASTp ${ }^{36}$ searches against the following databases: TIGRfam, Pfam, COG and NCBI-nr $\left(e\right.$-value $\left.<10^{-3}\right)$. To determine which ORFs from ANMV-1 genome share similarity to viral and prophage sequences, we compared our contig's translated ORFs with the ACLAME prophage-specific database ${ }^{37}$. To assess similarity to proteins from anaerobic methane-oxidizing archaea, we inspected NCBI-nr BLASTp results for ANME protein hits (uncultured archaeon, ANME-1; 'Candidatus Methanoperedens nitroreducens', ANME-2D; and uncultured archaeon, Gfoz37D1). A BLASTn survey was conducted against environmental metagenomic databases, including NCBI metagenomic sequences (env_nt), Moore Marine Virus Metagenomes ${ }^{30}$ and Pacific Ocean Virome sequences ${ }^{38}$, to find representatives sharing high nucleotide similarity (e-value $<10^{-20} ; 28$-nt word size) with ANMV-1.

The putative DGR TP of ANMV-1, AdtA, was analysed using Phyre2 (ref. 40) to find functional representatives based on secondary structural homology. Residues of TP that aligned with high confidence to the CLec fold region of the Mtd protein Bordetella phage BPP-1 (Phyre confidence $>90 \%$ ) were used to predict a three-dimensional model. Residue positioning was assessed by Ramachandran analysis and C-terminal variable residues were mapped from the primary sequence onto the predicted structure using Geneious v7.0 (Biomatters Ltd). 
Comparative analysis of Nanoarchaeota genomes. We identified DGR-like RTs via BLASTp searches against the NCBI-WGS database. For an initial proxy of DGR repeat features, we used the EMBOSS tool Dotmatcher ${ }^{40}$ to perform a dotplot analysis of homologous regions with moderate proximity ( $\pm 5 \mathrm{~kb}$ ) to RT. TR/VR regions were confirmed from candidates that comprised mostly adenine-specific variability, with at least 10 adenine-specific mismatches, with respect to one strand, and no more than 2 non-adenine mismatches in $100 \mathrm{bp}$ of aligned sequence.

DGR-containing sequences that were analysed in this study are from single-cell genomes belonging to DUSEL4 Nanoarchaeota, which were broadly described as part of a genome and metagenome annotation study on 'microbial dark matter' published elsewhere ${ }^{20}$. DUSEL4 Nanoarchaeota representatives were previously assigned into two OTUs comprising four single-cell genomes. We describe Nanoarchaeota DGRs with reference to their occurrence in combined single-cell sequence assemblies: OTU1 (genomes AAA011-G17 and AAA011-L22) and OTU2 (genomes AAA011-J02 and AAA011-K22). To confirm the presence of multiple distinct DGRs in one single-cell genome, we aligned OTU1 sequences with contigs from Nanoarchaeota AAA011-G17, which has the highest genome completeness of the DUSEL4 representatives ${ }^{20}$.

Nanoarchaeota RT sequences were aligned using ClustalW ${ }^{41}$ with sequences containing the catalytic RT domain, representing DGRs, group-II introns, retrons, long terminal repeats (LTRs), retroviruses, non-LTR elements and retroplasmids. The alignment was compared with a position-specific scoring matrix for the RVT-1 protein family (PF00078), and was manually realigned to conserve motifs considered essential for RT activity. Trees were constructed in MEGA v5.2 (ref. 42) using PhyML ${ }^{42}$ with the model LG + G + F. In addition, a PhyML tree was constructed from concatenated alignments of RT and TP amino-acid sequences to compare sequence similarities amongst Nanoarchaeota DGR cassettes.

TP expression and purification. Coding sequences of nanoarchaeal TPs were synthesized with codons optimal for expression in Escherichia coli (GENEWIZ, Inc.) and cloned into a modified $\mathrm{pET} 28 \mathrm{~b}$ expression vector with an $\mathrm{N}$-terminal His-tag followed by a PreScission protease cleavage site. Construct integrity was confirmed by DNA sequencing. TPs were expressed in Escherichia coli BL21-Gold (DE3) cells. Bacteria were grown with shaking at $37^{\circ} \mathrm{C}$ to an optical density (OD600) of $0.6-0.8$ and then cooled to room temperature, followed by induction with $0.5 \mathrm{mM}$ isopropyl $\beta-\mathrm{D}-1$-thiogalactopyranoside. Bacteria were grown with shaking at room temperature for $5-6 \mathrm{~h}$ further, then harvested by centrifugation $\left(25 \mathrm{~min}, 4,000 \mathrm{~g}, 4^{\circ} \mathrm{C}\right.$ ); the bacterial pellet was frozen at $-80^{\circ} \mathrm{C}$.

Cells were thawed and resuspended in buffer A $(300 \mathrm{mM} \mathrm{NaCl}, 50 \mathrm{mM}$ Tris (pH 8 ) and $5 \mathrm{mM} \beta$-mercaptoethanol; $20 \mathrm{mll}^{-1}$ of bacterial culture) supplemented with $1 \mathrm{mM}$ phenylmethylsulfonyl fluoride (PMSF). The bacteria were lysed by sonication and the lysate was centrifuged $\left(30 \mathrm{~min}, 35,000 \mathrm{~g}, 4^{\circ} \mathrm{C}\right)$. The following steps were performed at $4{ }^{\circ} \mathrm{C}$. The supernatant was applied to a column containing His-Select Nickel affinity gel (Sigma, $1 \mathrm{ml}$ of resin per $20 \mathrm{ml}$ of bacterial lysate), which had been equilibrated with buffer A. The column was washed with five column volumes of buffer B $(300 \mathrm{mM} \mathrm{NaCl}, 20 \mathrm{mM}$ Tris $(\mathrm{pH} 8)$ and $5 \mathrm{mM} \beta$ mercaptoethanol) containing $20 \mathrm{mM}$ imidazole, and the TP was eluted with buffer B containing $250 \mathrm{mM}$ imidazole. The His-tag was removed by PreScission protease cleavage (1:50 TP: protease mass ratio) overnight at $4{ }^{\circ} \mathrm{C}$. Cleaved TP was separated from non-cleaved proteins by applying the sample to a His-Select Nickel affinity gel column (Sigma) and collecting the flowthrough. The TP was further purified by gel filtration chromatography (Superdex 75) in $300 \mathrm{mM} \mathrm{NaCl}, 20 \mathrm{mM}$ Tris (pH 8) and $1 \mathrm{mM}$ dithiothreitol. Purified protein was concentrated to $2 \mathrm{mg} \mathrm{ml}^{-1}$ using ultrafiltration (10 kDa MWCO Amicon, Millipore); the concentration of TP was determined using a calculated molar extinction coefficient at $280 \mathrm{~nm}$ of $28,880 \mathrm{M}^{-1} \mathrm{~cm}^{-1}$.

CD spectroscopy. CD spectra were collected for the purified nanoarchaeal TP at $10 \mu \mathrm{M}$ in $300 \mathrm{mM} \mathrm{NaF}, 20 \mathrm{mM}$ sodium phosphate buffer, $\mathrm{pH} 8,1 \mathrm{mM}$ dithiothreitol on an Aviv $202 \mathrm{CD}$ spectrometer using a 1-mm pathlength cuvette. Spectra were recorded from 195 to $260 \mathrm{~nm}$ at $25^{\circ} \mathrm{C}$, with $1 \mathrm{~nm}$ wavelength steps and the measurement at each wavelength being averaged for $30 \mathrm{~s}$. A temperature melt study was carried out by increasing the temperature of the sample from 4 to $90^{\circ} \mathrm{C}$ in $1{ }^{\circ} \mathrm{C}$ increments, with the ellipticity being monitored at $216 \mathrm{~nm}$. The sample was then incubated at $90^{\circ} \mathrm{C}$ for $2 \mathrm{~min}$ and cooled from 90 to $4{ }^{\circ} \mathrm{C}$ in $1^{\circ} \mathrm{C}$ decrements, with the ellipticity being monitored at $216 \mathrm{~nm}$.

Tetranucleotide composition analysis. Tetranucleotide composition analysis can be used to identify core genome signatures to aid in taxonomic assignment, or to differentiate conserved protein-coding regions from those that were horizontally acquired $^{44-46}$. Tetranucleotide distributions of Nanoarchaeota genomes were determined as previously described ${ }^{43}$, using a custom Python script. Briefly, sequences were fragmented with a 5 -kb sliding window (500-bp overlapping step). Tetranucleotide frequencies were calculated by a zero-order Markov method, which applies odds ratios of observed counts for the 256 unique 4-mers, normalized to their respective mononucleotide frequencies. In order to assess tetranucleotide signatures for DGR regions ( $\sim 2 \mathrm{~kb}$ each), while avoiding a compositional bias of flanking sequence, we concatenated DGR cassettes from both OTU1 and OTU2 and fragmented this DGR-specific sequence $(\sim 21 \mathrm{~kb})$ with a sliding window as above. In addition, sequences from RT genes and TP genes were separately concatenated and fragmented with a sliding window as above to compare tetranucleotide compositions for the two DGR components.

Dimensionality reduction was performed via non-metric multidimensional scaling on Euclidean distances, using the vegan package in $\mathrm{R}^{47}$, and ordination ellipses representing the $95 \%$ confidence region were drawn with the 'ordiellipse()' function.

\section{References}

1. Kallmeyer, J., Pockalny, R., Adhikari, R. R., Smith, D. C. \& DHondt, S. Global distribution of microbial abundance and biomass in subseafloor sediment. Proc. Natl Acad. Sci. USA 109, 16213-16216 (2012).

2. Lipp, J., Morono, Y., Inagaki, F. \& Hinrichs, K.-U. Significant contribution of Archaea to extant biomass in marine subsurface sediments. Nature 454, 991-994 (2008).

3. Valentine, D. L. Adaptations to energy stress dictate the ecology and evolution of the Archaea. Nat. Rev. Microbiol. 5, 316-323 (2007).

4. Hoehler, T. M. \& Jørgensen, B. B. Microbial life under extreme energy limitation. Nat. Rev. Microbiol. 11, 83-94 (2013).

5. Lewin, A. et al. The microbial communities in two apparently physically separated deep subsurface oil reservoirs show extensive DNA sequence similarities. Environ. Microbiol. 16, 545-558 (2014).

6. Liu, M. et al. Reverse transcriptase-mediated tropism switching in Bordetella bacteriophage. Science 295, 2091-2094 (2002).

7. Doulatov, S. et al. Tropism switching in Bordetella bacteriophage defines a family of diversity-generating retroelements. Nature 431, 476-481 (2004).

8. Medhekar, B. \& Miller, J. F. Diversity-generating retroelements. Curr. Opin. Microbiol. 10, 388-395 (2007).

9. McMahon, S. A. et al. The C-type lectin fold as an evolutionary solution for massive sequence variation. Nat. Struct. Mol. Biol. 12, 886-892 (2005).

10. Guo, H. et al. Diversity-generating retroelement homing regenerates target sequences for repeated rounds of codon rewriting and protein diversification. Mol. Cell 31, 813-823 (2008).

11. Le Coq, J. \& Ghosh, P. Conservation of the C-type lectin fold for massive sequence variation in a Treponema diversity-generating retroelement. Proc. Natl Acad. Sci. USA 108, 14649-14653 (2011).

12. Rohwer, F. \& Vega Thurber, R. Viruses manipulate the marine environment Nature 459, 207-212 (2009).

13. Rowlands, T., Baumann, P. \& Jackson, S. P. The TATA-binding protein: a general transcription factor in eukaryotes and archaebacteria. Science 264, 1326-1329 (1994).

14. Dwivedi, B., Xue, B., Lundin, D., Edwards, R. A. \& Breitbart, M. A bioinformatic analysis of ribonucleotide reductase genes in phage genomes and metagenomes. BMC Evol. Biol. 13, 33 (2013).

15. Arambula, D. et al. Surface display of a massively variable lipoprotein by a Legionella diversity-generating retroelement. Proc. Natl Acad Sci. USA 110, 8212-8217 (2013).

16. Schillinger, T., Lisfi, M., Chi, J., Cullum, J. \& Zingler, N. Analysis of a comprehensive dataset of diversity generating retroelements generated by the program DiGReF. BMC Genomics 13, 430 (2012).

17. Goldrath, A. W. \& Bevan, M. J. Selecting and maintaining a diverse T-cell repertoire. Nature 402, 255-262 (1999).

18. Alder, M. N. et al. Diversity and function of adaptive immune receptors in a jawless vertebrate. Science 310, 1970-1973 (2005)

19. Stokke, R., Roalkvam, I., Lanzen, A., Haflidason, H. \& Steen, I. H. Integrated metagenomic and metaproteomic analyses of an ANME-1-dominated community in marine cold seep sediments. Environ. Microbiol. 14, 1333-1346 (2012).

20. Rinke, C. et al. Insights into the phylogeny and coding potential of microbial dark matter. Nature 499, 431-437 (2013)

21. Huber, H. et al. A new phylum of Archaea represented by a nanosized hyperthermophilic symbiont. Nature 417, 63-67 (2002).

22. Podar, M. et al. Insights into archaeal evolution and symbiosis from the genomes of a nanoarchaeon and its inferred crenarchaeal host from Obsidian Pool, Yellowstone National Park. Biol. Direct 8, 9 (2013).

23. Minot, S., Grunberg, S., Wu, G. D., Lewis, J. D. \& Bushman, F. D. Hypervariable loci in the human gut virome. Proc. Natl Acad. Sci. USA 109, 3962-3966 (2012).

24. Simon, D. M. \& Zimmerly, S. A diversity of uncharacterized reverse transcriptases in bacteria. Nucleic Acids Res. 36, 7219-7229 (2008).

25. Ye, Y. Identification of diversity-generating retroelements in human microbiomes. Int. J. Mol. Sci. 15, 14234-14246 (2014).

26. Louis-Jeune, C., Andrade-Navarro, M. A. \& Perez-Iratxeta, C. Prediction of protein secondary structure from circular dichroism using theoretically derived spectra. Proteins 80, 374-381 (2012).

27. Paull, C. K., Normark, W. R., Ussler, W., Caress, D. W. \& Keaten, R. Association among active seafloor deformation, mound formation, and gas hydrate growth and accumulation within the seafloor of the Santa Monica Basin, offshore California. Mar. Geol. 250, 258-275 (2008). 
28. Widdel, F. \& Bak, F. in: The Prokaryotes 2nd edn (eds Balows, A., Trüper, H. G., Dworkin, M., Harder, W. \& Schleifer, K.-H.) (Springer, 1992).

29. Thurber, R. V., Haynes, M., Breitbart, M., Wegley, L. \& Rohwer, F. Laboratory procedures to generate viral metagenomes. Nat. Protoc. 4, 470-483 (2009).

30. Henn, M. R. et al. Analysis of high-throughput sequencing and annotation strategies for phage genomes. PLoS ONE 5, e9083 (2010).

31. Schmieder, R., Lim, Y., Rohwer, F. \& Edwards, R. TagCleaner: identification and removal of tag sequences from genomic and metagenomic datasets. $B M C$ Bioinformatics 11, 341 (2010).

32. Hurwitz, B., Deng, L., Poulos, B. \& Sullivan, M. Evaluation of methods to concentrate and purify ocean virus communities through comparative, replicated metagenomics. Environ. Microbiol. 15, 1428-1440 (2013).

33. Niu, B., Fu, L., Sun, S. \& Li, W. Artificial and natural duplicates in pyrosequencing reads of metagenomic data. BMC Bioinformatics. 11, 187 (2010).

34. Sun, S. et al. Community cyberinfrastructure for advanced microbial ecology research and analysis: the CAMERA resource. Nucleic Acids Res. 39, D546-D551 (2011).

35. Delcher, A. L., Bratke, K. A., Powers, E. C. \& Salzberg, S. L. Identifying bacterial genes and endosymbiont DNA with Glimmer. Bioinformatics 23, 673-679 (2007).

36. Altschul, S. F., Gish, W., Miller, W., Myers, E. W. \& Lipman, D. J. Basic local alignment search tool. J. Mol. Biol. 215, 403-410 (1990).

37. Leplae, R., Hebrant, A., Wodak, S. J. \& Toussaint, A. ACLAME: a CLAssification of Mobile genetic Elements. Nucleic Acids Res. 32, D45-D49 (2004).

38. Hurwitz, B. L. \& Sullivan, M. B. The Pacific Ocean Virome (POV): a marine viral metagenomic dataset and associated protein clusters for quantitative viral ecology. PLoS ONE 8, e57355 (2013).

39. Kelley, L. A. \& Sternberg, M. J. Protein structure prediction on the Web: a case study using the Phyre server. Nat. Protoc. 4, 363-371 (2009).

40. Rice, P., Longden, I. \& Bleasby, A. EMBOSS: the European molecular biology open software suite. Trends Genet. 16, 276-277 (2000).

41. Larkin, M. A. et al. Clustal W and Clustal X version 2.0. Bioinformatics 23, 2947-2948 (2007)

42. Kumar, S., Nei, M., Dudley, J. \& Tamura, K. MEGA: a biologist-centric software for evolutionary analysis of DNA and protein sequences. Brief Bioinform. 9, 299-306 (2008).

43. Guindon, S. et al. New algorithms and methods to estimate maximumlikelihood phylogenies: assessing the performance of PhyML 3.0. Syst. Biol. 59, 307-321 (2010)

44. Pride, D. T., Meinersmann, R. J., Wassenaar, T. M. \& Blaser, M. J. Evolutionary implications of microbial genome tetranucleotide frequency biases. Genome Res. 13, 145-158 (2003).

45. Teeling, H., Meyerdierks, A., Bauer, M., Amann, R. \& Glöckner, F. O. Application of tetranucleotide frequencies for the assignment of genomic fragments. Environ. Microbiol. 6, 938-947 (2004).
46. Dick, G. J. et al. Community-wide analysis of microbial genome sequence signatures. Genome Biol. 10, R85 (2009).

47. Oksanen, J. et al. Vegan: Community Ecology Package. R package version 1.13-1 http://vegan.r-forge.r-project.org/ (2008).

\section{Acknowledgements}

This research was funded by National Science Foundation grant OCE-1046144 to D.L.V. and National Institutes of Health grant RO1 AI069838 to P.G. and J.F.M.; sequencing was provided through a Gordon and Betty Moore Foundation grant to the Broad Institute. We thank Tanja Woyke for assistance in examining Nanoarchaeota sequences from the Microbial Dark Matter project. For assistance with viral metagenome preparation and advice on bioinformatic analyses, we thank Steven Quistad and Rob Edwards. Yanling Wang provided helpful comments on an earlier draft of the manuscript.

\section{Author contributions}

B.G.P. performed the sediment incubations and purified viral DNA. B.G.P. and S.C.B. performed preprocessing and annotation of the metagenomic data set. B.G.P., S.C.B., E.C., D.A., S.H., A.S., P.G., J.F.M. and D.L.V conducted bioinformatic analyses of DGR sequences. S.H. and P.G. expressed and assayed nanoarchaeal target proteins and analysed the resulting data. B.G.P., S.C.B. and D.L.V. wrote the manuscript.

\section{Additional information}

Accession codes: Metagenomic sequence reads have been deposited in the NCBI BioSample database with accession code PRJNA47435.DV-ANM1. The ANMV-1 assembled genome sequence has been deposited in the NCBI nucleotide database with the accession code KP703175.

Supplementary Information accompanies this paper at http://www.nature.com/ naturecommunications

Competing financial interests: J.F.M. is a cofounder, equity holder and chair of the scientific advisory board of AvidBiotics Inc., a biotherapeutics company in San Francisco. The remaining authors declare no competing financial interests.

Reprints and permission information is available online at http://npg.nature.com/ reprintsandpermissions/

How to cite this article: Paul, B. G. et al. Targeted diversity generation by intraterrestria archaea and archaeal viruses. Nat. Commun. 6:6585 doi: 10.1038/ncomms7585 (2015).

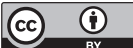

This work is licensed under a Creative Commons Attribution 4.0 International License. The images or other third party material in this article are included in the article's Creative Commons license, unless indicated otherwise in the credit line; if the material is not included under the Creative Commons license, users will need to obtain permission from the license holder to reproduce the material. To view a copy of this license, visit http://creativecommons.org/licenses/by/4.0/ 